\title{
Numerical self-consistent stellar models of thin disks
}

\author{
M. Ujevic and P. S. Letelier
}

Departamento de Matemática Aplicada, Instituto de Matemática, Estatística e Computação Científica, Universidade Estadual de Campinas, 13081-970 Campinas, SP, Brasil

e-mail: [mujevic;letelier]@ime.unicamp.br

Received 1 April 2005 / Accepted 13 July 2005

ABSTRACT

We find a numerical self-consistent stellar model by finding the distribution function of a thin disk that satisfies simultaneously the FokkerPlanck and Poisson equations. The solution of the Fokker-Planck equation is found by a direct numerical solver using finite differences and a variation of Stone's method. The collision term in the Fokker-Planck equation is found using the local approximation and the Rosenbluth potentials. The resulting diffusion coefficients are explicitly evaluated using a Maxwellian distribution for the field stars. As a paradigmatic example, we apply the numerical formalism to find the distribution function of a Kuzmin-Toomre thin disk. This example is studied in some detail showing that the method applies to a large family of actual galaxies.

Key words. stellar dynamics - methods: numerical - galaxies: general

\section{Introduction}

Few exact analytic stationary axisymmetric solutions for the collisionless Boltzmann equation are known in astrophysics. Most of the solutions correspond to spherical systems such as the polytropes and Plummer's models (Schuster 1883; Plummer 1911; Chandrasekhar 1939), and lowered isothermal models known as King models (Michie 1963; Michie \& Bodenheimer 1963; King 1966) which describe well globular cluster data. Also, we can find self-consistent spherical models by using Eddington's formula (Eddington 1916). Furthermore, at least half of spiral galaxies and most of the elliptical galaxies appear to be triaxial stellar systems. Models for triaxial systems have been constructed numerically, usually end products of $N$-body collapse simulations (Aarseth \& Binney 1978; Miller \& Smith 1979a,b; van Albada 1982; Wilkinson \& James 1982) or using catalogs of numerically integrated orbits (Schwarzschild 1979; Statler 1987; Teuben 1987); and analytically for special cases (Freeman 1966; Vandervoort 1980). Moreover, other self-consistent triaxial models that satisfy the Stäckel potentials were studied (de Zeeuw 1985; de Zeeuw et al. 1987; Hunter \& de Zeeuw 1992). Recently, a general solution of the Jeans equation for triaxial systems has been found (van de Ven et al. 2003). But, in many astrophysical objects light is emitted by a thin stellar disk, so planar galaxies are of more than academic interest. The most important exact analytical solutions of the collisionless Boltzmann equation for thin disks are the ones due to Mestel (1963) and Kalnajs (1972). Also, Miyamoto (1971) found an approximated analytical solution for the Toomre disk using a series method, and $\mathrm{Ng}$ (1967) found a self-consistent model for a thin stellar system, from a given distribution function, performing numerical calculations. The importance of having stationary solutions, for stellar objects or other systems, of the Boltzmann or Fokker-Planck equations is that they are a starting point for doing dynamical evolutions, and to study, analytically or numerically, the stability of the system by performing different kinds of perturbations. These studies have been done over the years by many authors, as examples we can mention (Cohn 1979; Watanabe et al. 1981; Nishida et al. 1984; Yepes \& Domínguez-Tenreiro 1992; Takahashi 1995; Theuns 1996; Takahashi et al. 1997; Joshi et al. 2001; Ashurov 2004). In most of the thin disk models, we only have as information their Newtonian gravitational potentials and surface mass density, knowing its distribution function we can obtain the thermodynamic variables allowing a full understanding of the physics of each individual model. Thus, the solution of the Fokker-Planck equation for each particular disk is needed to determine its main physical properties. Despite of the importance of the Kalnajs and Mestel thin disks, we have to keep in mind that they do not model a realistic physical situation because they exhibit odd behaviors within the disk, i.e. in the Kalnajs disks, the distribution function $f$ goes to infinity for certain values of the energy density and angular momentum; and in the Mestel disks, the gravitational potential $\Phi$ diverge at the center of the disk.

The Fokker-Planck equation is also known as the FokkerPlanck approximation because it truncates the BBGKY (Bogoliubov, Born, Green, Kirkwood, and Yvon) hierarchy of kinetic equations, at its lowest order, by assuming that correlation between particles only plays a role as a sequence of uncorrelated two-body encounters. It is worth noticing that the only 
"approximation" made in the Fokker-Planck equation comes from the model adopted for collisions and, in fact, the FokkerPlanck equation with a general collision term can be derived from first principles and no ad hoc suppositions are needed. The main goal of our work is to find and study a self-consistent model of a stellar thin disk, i.e. a distribution that satisfies simultaneously the Fokker-Planck and the Poisson equation, the meaning of simultaneously will become clear later in the manuscript. In writing the Fokker-Planck equation, we considered in the collision term the local approximation and used the diffusion coefficients found by Rosenbluth et al. (1957).

The computational problems in solving the Fokker-Planck equation without using any statistical (Monte Carlo method) and average (moment equations) are briefly discussed in this paper. The Fokker-Planck equation is solved by a direct numerical solver using finite differences and a variation of Stone's method (Stone 1968; Ujevic \& Letelier 2005) to include mixed derivatives. This allows high resolution solutions with lower computational cost (Ujevic \& Letelier 2005). Due to nature of the problem, either analytical or numerical solutions are very difficult to obtain. In this respect, it is illustrative to cite the words of Binney \& Tremaine (1987, page 245): Finding the particular function of three variables that describes any given galaxy is no simple matter. In fact, this task has proved so daunting that only in the last few years, three-quarters of a century after Jeans's (Jeans 1915) paper posed the problem, has the serious quest for the distribution function of even our own Galaxy got underway. As far as we know, there are no analytical or numerical solution of the Fokker-Planck equation for thin disks.

The article is organized as follows. In Sect. 2 we present the Fokker-Planck equation for a thin disk with the collision term given by the local approximation and the diffusion coefficients found by Rosenbluth et al. (1957). In Sect. 3, we explain briefly the computational codes used to solve the FokkerPlanck equation. In Sect. 4, we present the properties of the Kuzmin-Toomre thin disk used as an example for the numerical method. The numerical results for the velocity and spatial distribution functions for galaxies based in this model are showed. Finally, in Sect. 5, we summarized our results.

\section{Fokker-Planck equation for a thin disk}

The general problem for the evolution of a system made with stars of mass $m$ using the Fokker-Planck equation consists in the determination of a distribution function $f$ and the gravitational potential $\Phi$ that solve the Fokker-Planck and Poisson equations, say

$$
\begin{aligned}
& \frac{\partial f}{\partial t}+\boldsymbol{v} \cdot \nabla f-\nabla \Phi \cdot \nabla_{v} f=\Gamma[f], \\
& \nabla^{2} \Phi=4 \pi G m \int f \mathrm{~d}^{3} v,
\end{aligned}
$$

where $v$ represents the velocity of the stars, $\nabla$ is the usual spatial gradient, $\nabla_{v}$ is the velocity gradient (derivations are done with respect to the velocities), $G$ is the gravitational constant and the symbol $\Gamma[f]$ represents the Fokker-Planck collision term. The solution of the Fokker-Planck and Poisson equations is found from an iterative procedure in an short time interval with respect to the time scale in which $\Phi$ varies. The method of evolution has two main steps: (i) the distribution function is first advanced in time using Eq. (1) with the potential held fixed, and (ii) the potential is then adjusted so that the Poisson equation is again satisfied. Some systems, as a final state of the evolution, may reach a stationary regime. Then, the temporal part in (1) is equal zero and the distribution function is time independent. Hence, in this case, the distribution function must satisfy simultaneously the stationary Fokker-Planck equation

$\boldsymbol{v} \cdot \nabla f-\nabla \Phi \cdot \nabla_{v} f=\Gamma[f]$,

together with Eq. (2). Numerically we have that, in a nonstationary regime, a gravitational potential at time $t$ creates through the Fokker-Planck equation a distribution function at a later time $t+\Delta t$. In our case, a stationary regime, we want to find a gravitational potential and a distribution function at the same time $t$. This is what we mean when we say that the solution $(\Phi, f)$ satisfies simultaneously the Fokker-Planck and Poisson equation. Note that we can start with a given gravitational potential at time $t$ and make the evolution of the system by first finding its distribution function at a time $t+\Delta t$, and later, the new gravitational potential, via Eq. (2), at this new time $t+\Delta t$, and hope that the successive iterations of this procedure converge. In the case of convergence, we obtain also a stationary solution but this solution might not have any physical significance, i.e. the final gravitational potential together with the distribution function do not model any astrophysical system. For that reason, it is important to find the simultaneous stationary solution of the Fokker-Planck equation for a given gravitational potential that can describe a real system. The procedure to find this solution is explained in the next section. Our main goal is to obtain a physical consistent model by solving the set of Eqs. (2), (3) without using any approximations, like the ones found in the orbit averaged approximation, Monte Carlo method, or the Jeans equations (Binney \& Tremaine 1987), i.e. a direct numerical solution.

To find the stationary Fokker-Planck equation valid for thin disks, the non-stationary case is straightforward, we start from the general case in three dimensions and later perform the projection to the thin disk plane. The collision term in the local approximation has the form

$$
\begin{aligned}
\Gamma[f]= & -\sum_{i=1}^{3} \frac{\partial}{\partial v_{i}}\left[f(\boldsymbol{x}, \boldsymbol{v}) D\left(\Delta v_{i}\right)\right] \\
& +\frac{1}{2} \sum_{i, j=1}^{3} \frac{\partial^{2}}{\partial v_{i} \partial v_{j}}\left[f(\boldsymbol{x}, \boldsymbol{v}) D\left(\Delta v_{i} \Delta v_{j}\right)\right],
\end{aligned}
$$

where the functions $D\left(\Delta v_{i}\right)$ and $D\left(\Delta v_{i} \Delta v_{j}\right)$ are known as the diffusion coefficients. These coefficients were calculated (Rosenbluth et al. 1957) assuming and inverse square force for the interaction, and also, that each stellar encounter involve only a single pair of stars and are independent of all others. They are simplified if the field stars distribution function is a Maxwellian distribution (Binney \& Tremaine 1987). Recall that the diffusion coefficients are calculated considering a test star of mass $m$ moving through an infinite homogeneous sea 
of field stars of mass $m_{a}$ who has mean velocity equal to zero. We know from stabilities analysis that this gravitating system can not be in static equilibrium and to overcome this problem we invoke the Jeans swindle. In order to calculate the diffusion coefficients in the next sections, we are going to set the dispersion of the field stars equal to the typical velocity of the problem considered.

The diffusion coefficients described above are valid for a general three dimensional case. To obtain the Fokker-Planck equation for thin disks, we perform a projection on the $z=0$ and $v_{z}=0$ planes using Dirac deltas. Doing the replacement

$f\left(x, y, z, v_{x}, v_{y}, v_{z}\right) \rightarrow f\left(x, y, z, v_{x}, v_{y}, v_{z}\right) \delta(z) \delta\left(v_{z}\right)$,

in (1), and integrating in the $z$ and $v_{z}$ variables, we arrive at a stationary Fokker-Planck equation in two dimensions, see Appendix A. The indexes $(i, j)$ of the collision term (4) now take only the values 1 and 2 . The important thing about this procedure is that the form of the Fokker-Planck equation and the diffusion coefficients are preserved but projected into the $z=0$ and $v_{z}=0$ planes.

\section{Numerical method}

The solution of the Fokker-Planck equation in a stationary regime is not an easy task. The problems involve in the solution are both numerical and physical. Numerical because in the three dimensional case, the Fokker-Planck equation has six independent variables, three space coordinates $(\boldsymbol{x})$ and three velocity coordinates $(\boldsymbol{v})$. In the two dimensional case, the disk case, a simplification exist because the total number of variables are four. In either cases, in a finite difference scheme, the large number of grid nodes needed for the computation of the solution becomes a data storage problem. In a three dimensional problem, if we divided each of the six variables intervals of the distribution function in nine parts (ten nodes), we will have a grid with $10^{6}$ nodes. This corresponds to a same number of linear equations to solve. In a simple numerical method we have to store and solve a matrix with $10^{12}$ elements. For two dimensional disks, the main matrix will have $10^{8}$ elements. So, our problem is slightly simpler than the three dimensional case. With 10 grid nodes per variable only very simple geometries can be described. Furthermore, the large number of matrix elements brings us another computational problem, the slowness of the codes. Most of the elements of the these matrices are zero and this motivates the search for alternative and faster methods to solve the linear system using only the non null data. We overcome these problems performing a discretization of the Fokker-Planck equation using a finite difference thirteen-point molecule method and then solving the resulting discretization matrix with a modification of the method proposed by Stone (1968). The original method of Stone was modified to handle two (four variables) and three (six variables) dimensions, and the extra diagonals that appear when mixed derivatives are used (Ujevic \& Letelier 2005). Also, it can handle nonstationary problems, and curve boundary conditions with a little modification of the code. Remember that the collision term in the Fokker-Planck equation has mixed derivatives. Physical because the search for a boundary condition is not always clear.
In general, the analytic potentials that we have are only geometrical, i.e they have been developed without using any observational data (only an image method solution for a point mass). From this fact, it is not obvious that these potentials could represent some real galaxies.

The surface mass density of the disk is obtained integrating the right hand side of (2), say

$\Sigma=4 \pi G m \int_{-\infty}^{\infty} f \mathrm{~d}^{3} v$

Due to the fact that the integral limits of (6) are from $-\infty$ to $\infty$, we must define first a criteria to establish the velocity domains for the calculation of the distribution function. In general, we are going to use the value of the escape velocity $v_{\text {esc }}=\sqrt{2|\Phi|}$ at the center of the disk to establish the upper and lower limits of the integration.

When Cartesian coordinates are used, the spatial disk part of the distribution function will be delimited by an irregular boundary (circumference). By using cylindrical coordinates we can transform the irregular domain into a more simple square domain, but in this case we need more boundary conditions and assumptions for the distribution function. For example, if we are using a Dirichlet boundary conditions, we need the value of the distribution function on the side of the square that corresponds to the coordinate $R=\sqrt{x^{2}+y^{2}}=0$ (the center of the thin disk), a value that is not always known. To avoid these difficulties, we prefer to use Cartesian coordinates in the spatial domain and treat the problem of the irregular boundary, the code presented in (Ujevic \& Letelier 2005) allows this option. In compensation, we only need the value of $f$ in the exterior radius of the disk, i.e. only one expression for the boundary condition.

The integration program was tested by integrating exponential functions of the form $\exp \left[\left(-v_{x}^{2}-v_{y}^{2}\right) / 2 \sigma^{2}\right]$ for different values of $\sigma$. The integral is performed using Romberg's integration scheme with Richardson style extrapolation (Gerald $\&$ Wheatley 1994). The Fokker-Planck code was tested comparing the results obtained with the modified Stone method with other methods, as for example, the explicit Euler method and Gauss elimination, obtaining full agreement. This was performed for a small number of nodes because for larger grids the usual methods are not convenient. All these tests assure us that our codes are working properly. The $L U$ decomposition used in the modified Stone method is presented in the Appendix B.

The general procedure to find the distribution function of a thin disk is as follows: first we start with a given stationary disk potential which is inserted into the Fokker-Planck equation. Then, we find numerically a distribution function in phase space associated to the gravitational potential and the boundary condition imposed. Later, we perform the integration (6) using the recently calculated distribution function to find the surface mass density of the problem. This surface mass density has to be equal to the surface mass density of the given gravitational potential if we want to have stationary self-consistent solutions between the Fokker-Planck and Poisson equations. If the surface mass density obtained after the integration of (6) is not equal to the one given a priori then it is an indication that the boundary condition used for the distribution function is not 
compatible with the problem studied. Our goal is to find the self-consistent solution of the problem by adjusting the parameters involve in the gravitational potential, galaxy model and boundary condition of the Fokker-Planck equation. Note, that in this procedure we do not solve the Laplace equation. This was done only for didactic reasons and because there exist in the literature a huge variety of methods that solve this equation. Actually, to obtain a self-consistent model, we only need the surface density of the thin disk. With this information, we find the gravitational potential via Laplace equation, and later we apply the above procedure.

Now, we need a boundary condition for $f$. An appropriate boundary condition can be found if we recall that the velocity distribution in a star system is determined by two competing processes: relaxation through stellar encounters that drives the distribution toward a Maxwellian form, but stars beyond the finite escape velocity continually disappear from the system. Since escaping stars traverse the system in a small fraction of a relaxation time, the resultant velocity distribution should be zero beyond the escape velocity but otherwise nearly Maxwellian. Also, most of the stars in thin galaxies are centrally concentrated. So, for a stationary system, these considerations lead us to use as an appropriate boundary condition for our disk the function

$f_{\text {Boun }} \propto \exp \left[\left(-x^{2}-y^{2}\right) / 2 \sigma_{\mathrm{s}}^{2}\right] \exp \left[-\left(v-v_{\text {typ }}\right)^{2} / 2 \sigma_{\mathrm{v}}^{2}\right]$,

where $\sigma_{\mathrm{s}}$ and $\sigma_{v}$ are the spatial and velocity dispersion respectively, and $v=\sqrt{v_{x}^{2}+v_{y}^{2}}$. The velocity dispersion $\sigma_{v}$ is a measure of the deviation from the star typical velocity of the galaxy. The spatial dispersion $\sigma_{\mathrm{s}}$ is a measure of how centrally concentrated these stars will be. The velocity part of the boundary condition is centered at the typical velocity of the system.

\section{Kuzmin-Toomre galactic model}

The code used, and the procedure presented in this manuscript, can be applied, in principle, to find the distribution function for any thin disk potentials. But, as an example, we are going to use the Kuzmin-Toomre family of thin disks (Kuzmin 1956; Toomre 1963; Binney \& Tremaine 1987) which, in cylindrical coordinates, has the Newtonian gravitational potential,

$\Phi_{\mathrm{TK}}(R, z)=-\frac{G M}{\sqrt{R^{2}+(a+|z|)^{2}}}$.

When $z<0, \Phi_{\mathrm{TK}}$ coincides with the potential of a point mass $M$ located at the point $(R, z)=(0, a)$; and when $z>0$, $\Phi_{\mathrm{TK}}$ is exactly the potential of a point mass at $(0,-a)$, hereafter $a$ is denoted as the cut parameter. The cut parameter modifies physical properties of the disk, like energy density, pressures and sound velocity profiles (Vogt \& Letelier 2003). By applying Gauss's theorem to a flat volume that contains a small portion of the plane $z=0$, we conclude that the potential (8) is generated by the surface density

$\Sigma_{\mathrm{TK}}(R)=\frac{a M}{2 \pi\left(R^{2}+a^{2}\right)^{\frac{3}{2}}}$.

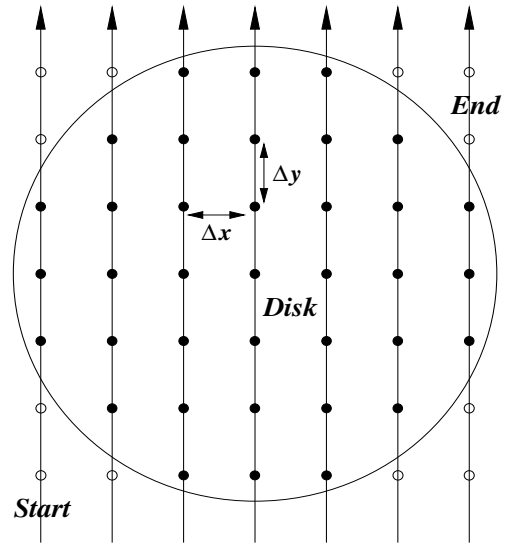

Fig. 1. Nodes in the spatial domain to be used in the two dimensional figure of the surface mass density. The full circles represents the nodes inside the disk used for the calculation. The empty circles are necessary for maintaining an order in the grid but do not enter into the solution. After finding the surface mass density, the spatial part of the solution of the distribution function can be divided into vectors (represented like arrows). This procedure help us to present the surface mass density results in a simple way using a two dimensional figure.

The potential (8) describes a disk which extends from $R=0$ to $R=\infty$. In this work we shall consider a disk with finite radius with the same type of potential.

We start with a galaxy model of radius $40 \mathrm{kpc}$ containing $10^{12}$ stars of solar masses $M_{\odot}$. The typical streaming velocity considered is of order $200 \mathrm{~km} \mathrm{~s}^{-1}$ with velocity dispersion $\sigma_{v}=40 \mathrm{~km} \mathrm{~s}^{-1}$. Hereafter, we set the masses of the field and test stars equal $\left(m=m_{a}=M_{\odot}\right)$. The mass density $\rho$ that appears in the diffusion coefficients is calculated spreading the total mass of the system in a thin disk, which radius is given by the model. To solve the Fokker-Planck equation for the KuzminToomre disk type potential we use a total of 810000 nodes, $30^{4}$, distributed along the computational domain. All the nodes are necessary to maintain a computational order that is needed by the code, but actually we do not used all of these nodes in the calculation because some of them lie outside the thin disk region, see Fig. 1. Recalling that, we have to check the results of our numerical solution of the distribution function indirectly, we compare the surface mass density obtained from the integral (6) with the exact solution (9). This will give us an estimate of how far we are from the exact distribution function. In Fig. 1 we present the spatial part of a disk with a given number of nodes used for the computation.

For every internal node of the spatial part (full circles in Fig. 1) we perform the integration of the distribution function (6) obtaining in that way the surface mass density of the disk. Note that the surface density is only known in these internal nodes. This surface mass density can be plotted in a conventional three dimensional plot $(x, y, \Sigma)$. But, this makes the visual comparison with the exact result $\Sigma_{\mathrm{TK}}$ cumbersome. For ease in plotting and comparison, we shall plot the surface mass density in a two dimensional plot $(\{x, y\}, \Sigma)$. The nodes $\{x, y\}$ are chosen following the direction of the arrows, starting from the left start arrow. Once finished, we moved to the next right arrow and plot the $\{x, y\}$ nodes of this arrow along the nodes of 
the previous arrow. We repeat this procedure until we reach the end arrow. The nodes that are outside the disk (empty circles) have surface mass density equal to zero and will be omitted for the plot. We use this procedure for the numerical solution as well as for the exact mass surface density solution. In the later case, we take the exact values corresponding to the grid nodes used in the numerical solution. We show the results in a two dimensional figure, instead of a three dimensional one, because we think that the value difference of the mass surface density between the grid points in the numerical and exact solutions can be noted easily. The spatial difference between arrows is the spatial discretization $\Delta x$ used in the solution of the Fokker-Planck equation.

In the upper graph of Fig. 2 we present the numerical result of the mass surface density (6) with parameters $\sigma_{\mathrm{s}}=$ $35 \mathrm{kpc}, \sigma_{v}=40 \mathrm{~km} \mathrm{~s}^{-1}$ and $a=40 \mathrm{kpc}$ (dotted line), compared with the exact density function (solid line) obtain from the data of the Kuzmin-Toomre density function (9). In this case $v_{\mathrm{esc}} \approx 460 \mathrm{~km} \mathrm{~s}^{-1}$ and the integration have been performed in the intervals $v_{x} \in[-350,350] \mathrm{km} \mathrm{s}^{-1}$ and $v_{y} \in$ $[-350,350] \mathrm{km} \mathrm{s}^{-1}$. By varying the cut parameter $a$ and the spatial dispersion $\sigma_{\mathrm{s}}$, we can lower or raise the height of the ridges in the computed curves. note that this modifications also leads to changes in the escape velocity and integration intervals. In the lower graph of Fig. 2 we show the case with parameters $\sigma_{\mathrm{s}}=40 \mathrm{kpc}, \sigma_{v}=40 \mathrm{~km} \mathrm{~s}^{-1}$ and $a=62.7 \mathrm{kpc}$. For these parameters, $v_{\mathrm{esc}} \approx 370 \mathrm{~km} \mathrm{~s}^{-1}, v_{x} \in[-250,250] \mathrm{km} \mathrm{s}^{-1}$ and $v_{y} \in[-250,250] \mathrm{km} \mathrm{s}^{-1}$. The maximum relative error between the computed values and the exact values is in this case less than $1 \%$. This is a good result because we are trying to match a real finite physical galaxy to an infinite geometric potential that was developed without using any observational data. In solving the Fokker-Planck equation we must take into account that the final numerical result of the distribution function has to be positive. Numerically, this means that all the values of the distribution function at the grid nodes have to be positive. Numerical simulations show us that this leads to restrictions in the velocity dispersion and velocity intervals. For example, if we lowered the values for the velocity dispersion to values near zero, the distribution profile becomes very sharp and then some of the values of the distribution function are negatives. The value of the velocity dispersion depends on the galaxy model under consideration. In particular, for the solution found above, we can not say that the distribution function is unique because it depends on the relative error allowed for the numerical solution. For example, if we allowed a relative error of $1.5 \%$, the parameters $a$ and $\sigma_{\mathrm{s}}$ can take values between [62.4 kpc, $\left.63 \mathrm{kpc}\right]$ and [39.9 kpc, $40.1 \mathrm{kpc}$ ] respectively; and $\sigma_{v}$ can not take values lower than $25 \mathrm{~km} \mathrm{~s}^{-1}$ (because some values of the distribution function are negatives) but might have higher values. In general, for the gravitational potential (8), we were able to model galaxies with positive distribution functions for different parameters values, i.e. galaxies with total number of stars ranging from $10^{10}$ to $10^{12}$, typical velocities from $100 \mathrm{~km} \mathrm{~s}^{-1}$ to 300 $\mathrm{km} \mathrm{s}^{-1}$, velocity dispersions from $20 \mathrm{~km} \mathrm{~s}^{-1}$ to $80 \mathrm{~km} \mathrm{~s}^{-1}$ and galaxies radius from $10 \mathrm{kpc}$ to $80 \mathrm{kpc}$. So, in principle, we can find stationary solutions for many typical galaxies.
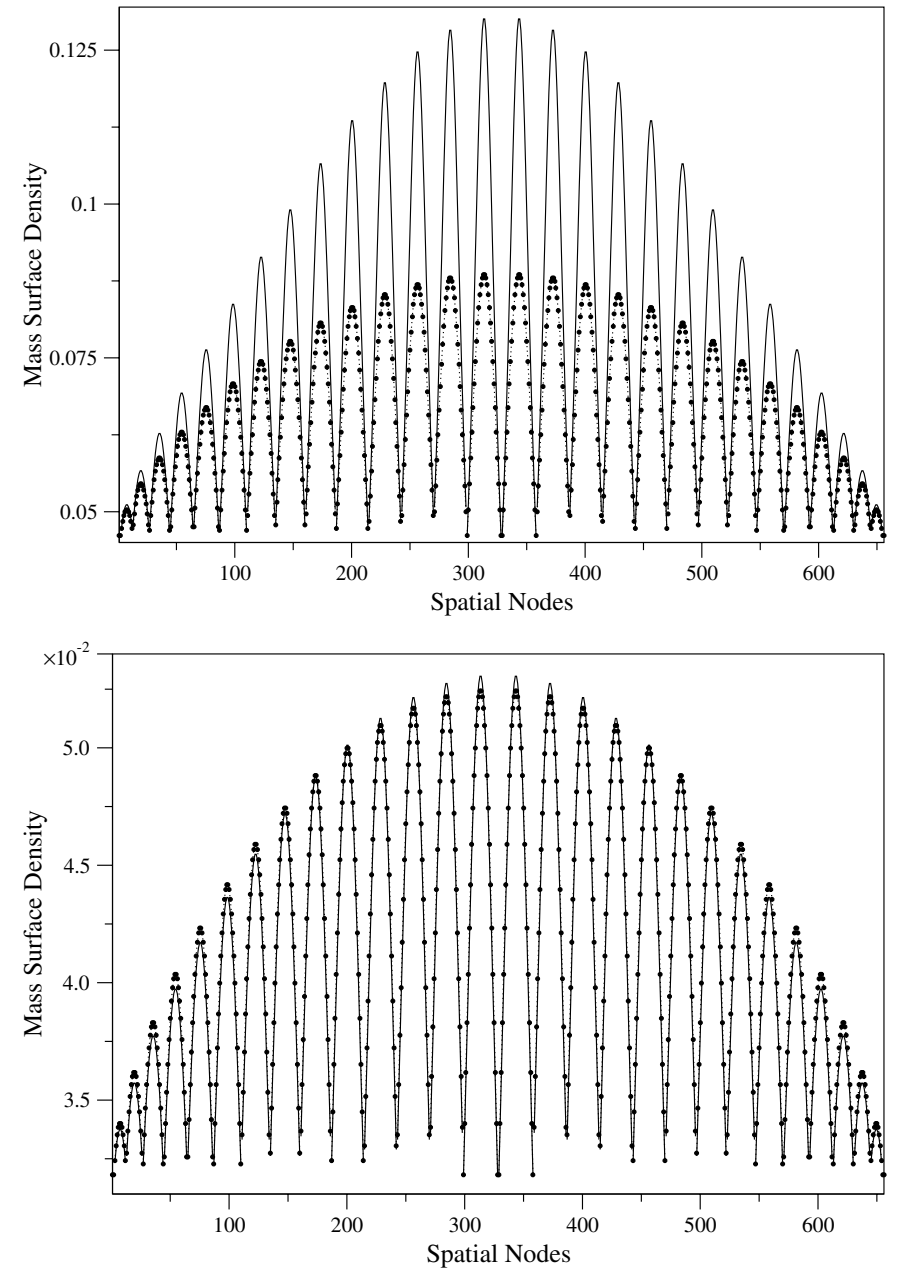

Fig. 2. Top: two dimensional plot of the numerical solution for the mass surface density of the Kuzmin-Toomre like disk with cut parameter $a=40 \mathrm{kpc}$, and $\sigma_{\mathrm{s}}=35 \mathrm{kpc}$. The solid line is the exact solution obtained from the mass surface density of the Kuzmin-Toomre disk type. The dotted line represents the mass surface density obtained after the integration of the numerical solution, the full circles are the values of the numerical calculations. The arrows from Fig. 1 are plotted one along the others. Each point represents a position $\{x, y\}$ on the surface of the disk. Bottom: the same of Top but with cut parameter $a=62.7 \mathrm{kpc}$ and $\sigma_{\mathrm{s}}=40 \mathrm{kpc}$. Adjusting the values of the cut parameter $a$ and the spatial dispersion $\sigma_{\mathrm{s}}$ we can approach the numerical mass surface density profile to the exact solution.

Note that the potential we are using is from an infinite thin disk, and that we have made a cut at an usual radius for galaxies. So, the results we obtain are remarkable because we modeled a stationary galaxy with physical parameters from a infinite-cut disk which do not necessarily describes a real galaxy. Better agreement can be found if we enlarge the value of the spatial dispersion above the fixed radius of the galaxy. This behavior is equal for every size of the galaxy we choose, a numerical indication that the galaxy is actually bigger than our chosen radius. This is not surprising because the gravitational potential used in the Fokker-Planck equation is originally from an infinite thin disk.

All the calculations have been done without actually knowing the proportionality constant appearing in (7). To determine 


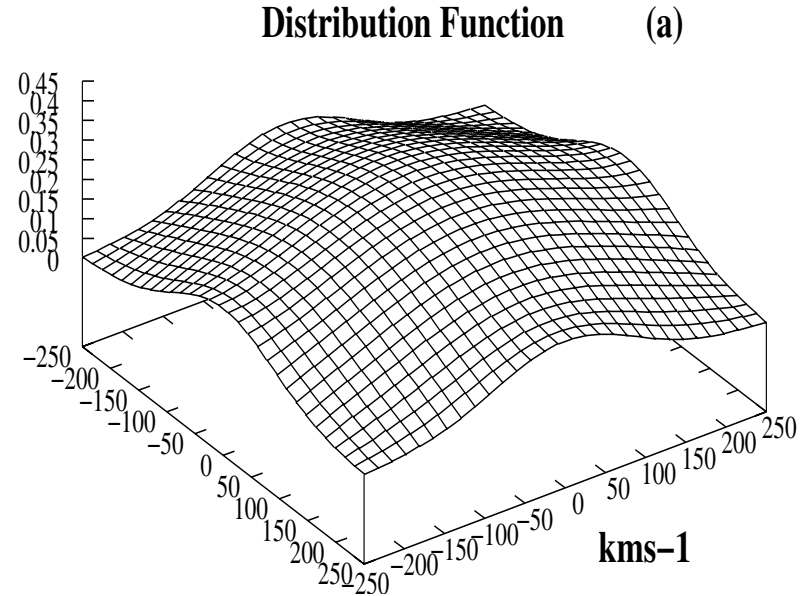

Distribution Function

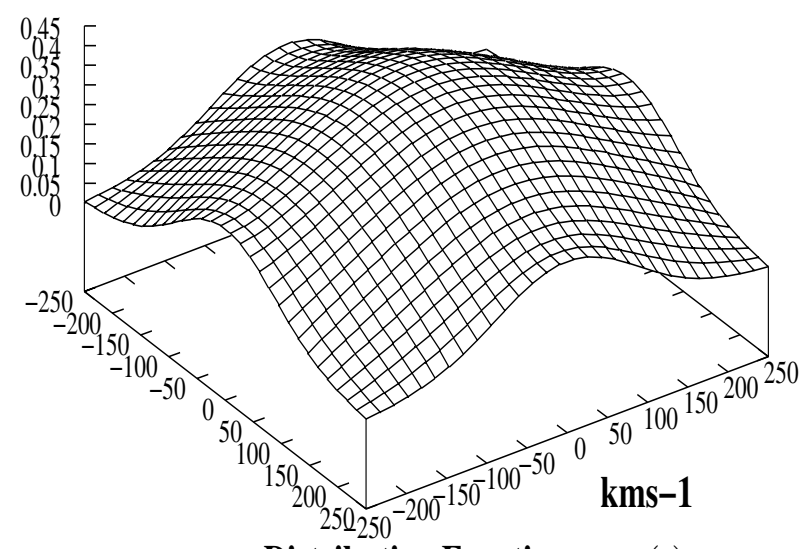

Distribution Function

(c)

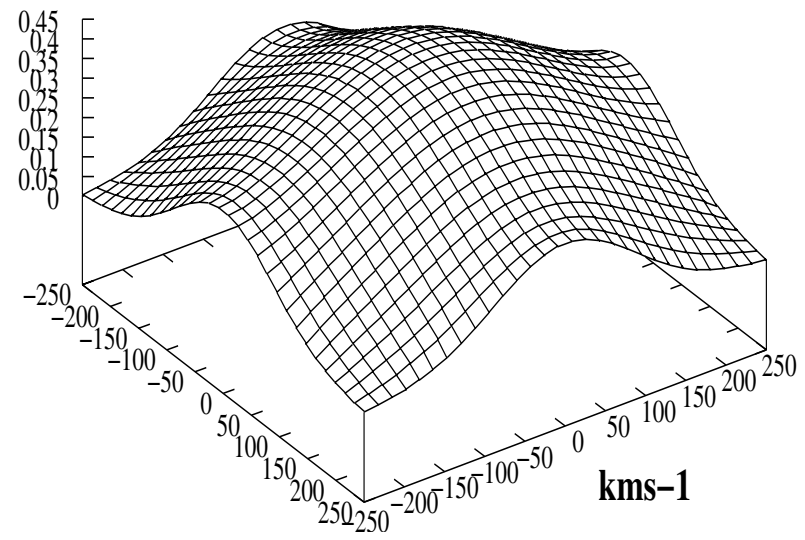

Fig. 3. Numerical solution of the velocity distribution of the distribution function for a Kuzmin-Toomre disk type when $a=62.7 \mathrm{kpc}$ and $\sigma_{\mathrm{s}}=40 \mathrm{kpc}$. The velocity distribution functions correspond to radius $r=39.26 \mathbf{a}), 20.37 \mathbf{b}), 1.95 \mathbf{c}) \mathrm{kpc}$.

the proportionality constant, we take one point of the initial data for the problem, the mass surface density $\Sigma_{\mathrm{TK}}(9)$, and the numerical mass surface solution at the same position. Later, the numerical solution is scaled with this constant.

The numerical solution of the distribution function obtained in the cases mentioned before can only be seen by a collection of three dimensional figures. For example, if we want the velocity distribution for the spatial part, we will have one figure for each pair $(x, y)$. In Fig. 3, we show the velocity
Distribution Function

(a)

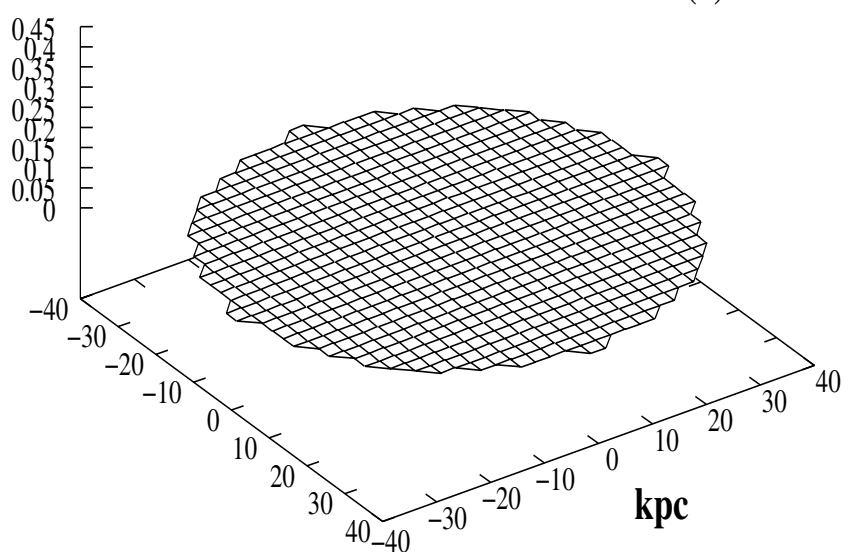

Distribution Function

(b)

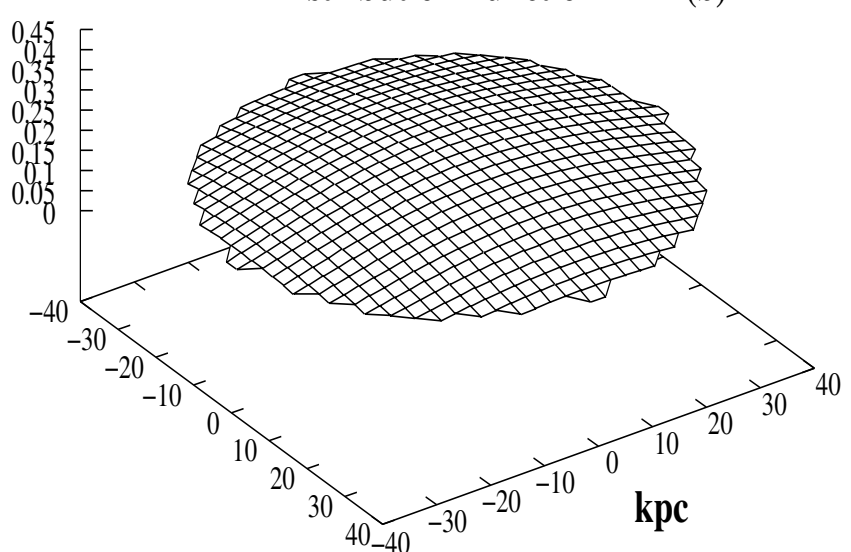

Distribution Function

(c)

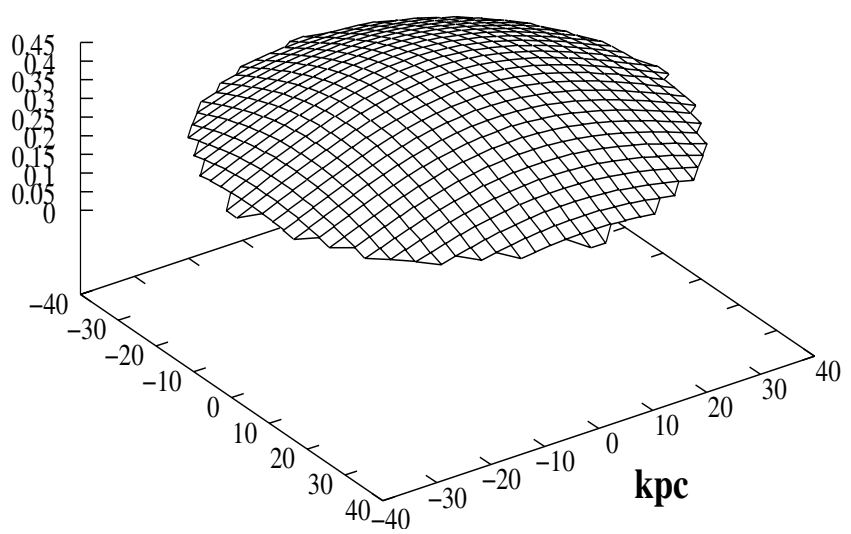

Fig. 4. Numerical solution of the spatial distribution of the distribution function for a Kuzmin-Toomre disk type when $a=62.7 \mathrm{kpc}$ and $\sigma_{\mathrm{s}}=40 \mathrm{kpc}$. The spatial distribution functions correspond to velocities $\left.\left.\left.v=\sqrt{v_{x}^{2}+v_{y}^{2}}=341.6 \mathbf{a}\right), 207.3 \mathbf{b}\right), 27.3 \mathbf{c}\right) \mathrm{km} \mathrm{s}^{-1}$.

distribution function at three different radius for a KuzminToomre thin disk with parameters $\sigma_{\mathrm{s}}=40 \mathrm{kpc}, \sigma_{v}=40 \mathrm{~km}$ $\mathrm{s}^{-1}$ and $a=62.7 \mathrm{kpc}$. From these figures we note that, in order to have a stationary galaxy, the velocity distribution function have to be centrally concentrated. In all the cases, the population of stars with velocities that are near the escape velocity of the system is almost equal to zero. Also, in Fig. 4 we present three spatial distribution functions for the model of Fig. 3. 
We note from these figures that in general the population of stars increases with lower velocities, and that for each particular velocity the concentration of stars are bigger near the center of the galaxy than in the edge of the galaxy. Note that the distribution function can have other symmetries besides the axially symmetry presented in the gravitational potential (8) and surface density (9) due to the form of Eq. (3).

Note that the distribution function obtained from (3) has to be positive and also, after the integration (6), the surface density profile obtained has to match the initially given surface density (9). These facts impose restrictions on the boundary conditions and for that reason the boundary condition can not be set arbitrarily. The boundary condition (7) used for the numerical calculation satisfies all the above requirements. We have tried other boundary conditions by simple modifying the exponents of Eq. (7) and with other different functions without obtaining good results.

\section{Conclusions}

We describe a method to find numerically a distribution function that simultaneously satisfies the Fokker-Planck and Poisson equations providing a self-consistent galaxy model for thin disks. This solution have been found by solving the original Fokker-Planck equation avoiding simplification using a previously developed code based on a direct finite differences method.

As an example, we find distributions function of the family of Kuzmin-Toomre thin disks. As far as we test, we are able to model many self-consistent stationary galaxies using the most common physical parameters known for galaxies. This is accomplished by adjusting the parameters found in the gravitational potential, galaxy model and boundary condition. In principle, we can find consistent astrophysical model for other thin disk potentials. Once the solution is found, the stability analysis of the models can be achieved performing perturbations to the stationary solutions previously found and using the same code presented in Ujevic \& Letelier (2005) and performing a Crank-Nicolson discretization in time. We also found that (7) is an appropriate boundary condition for the case of a stationary Kuzmin-Toomre stationary disk. In forthcoming articles this condition will be checked for other thin disk models as well as three dimensional disk models.

In this manuscript we presented the results for a case in which the gravitational potential and the surface density is known a priori. This was done only for didactic purposes because, in general, the method presented here can be applied to more general problems in which we only know the surface mass density of the disk. With this condition, the gravitational potential can be obtained, numerically most of the time, through the Poisson Eq. (2), and then the distribution function $f$ through the Fokker-Planck equation. Later with $f$, the complete surface density is found via the integral (6). Then, the problem is completely solved by matching the surface mass density obtained via the integral (6) with the one found from the Poisson equation.

For future applications, it will be very important to test this code and boundary condition in other thin disk models and three dimensional structures in order to calculate their distribution functions. Three dimensional models, can be obtained following the procedure of this manuscript together with the code in Ujevic \& Letelier (2005). In the last reference, a three dimensional calculation of a test Fokker-Planck equation is presented. The construction of a solution for Plummer and King models, thick disks, and the stability of thin disks and other three dimensional structures are currently under investigation by the authors. A stability analysis of several thin disks using a perturbation on its energy-momentum tensor in the context of general relativity can be found in Ujevic \& Letelier (2004), another stability analysis of related structures using Rayleigh criteria of stability can be found in Letelier (2003).

Acknowledgements. M.U. and P.S.L. thanks FAPESP for financial support and P.S.L. also thanks CNPq.

\section{Appendix A: Projection into the $z-V_{z}$ plane}

In this section, we develop some of the calculations that we encounter in the substitution of the modified distribution function (5) into the stationary Fokker-Planck Eq. (3),

$$
\begin{gathered}
\iint \frac{\partial}{\partial z}\left[f \delta(z) \delta\left(v_{z}\right)\right] \mathrm{d} z \mathrm{~d} v_{z}=\int\left[\frac{\partial f}{\partial z} \delta(z)+f \dot{\delta}(z)\right] \mathrm{d} z \\
=\left.\frac{\partial f}{\partial z}\right|_{z=0}-\left.\frac{\partial f}{\partial z}\right|_{z=0}=0, \\
\iint \frac{\partial}{\partial v_{z}}\left[f D\left(\Delta v_{z}\right) \delta(z) \delta\left(v_{z}\right)\right] \mathrm{d} z \mathrm{~d} v_{z} \\
=\int\left\{\frac{\partial}{\partial v_{z}}\left[f D\left(\Delta v_{z}\right)\right] \delta\left(v_{z}\right)+f D\left(\Delta v_{z}\right) \dot{\delta}\left(v_{z}\right)\right\} \mathrm{d} v_{z} \\
=\left.\frac{\partial}{\partial v_{z}}\left[f D\left(\Delta v_{z}\right)\right]\right|_{v_{z}=0}-\left.\frac{\partial}{\partial v_{z}}\left[f D\left(\Delta v_{z}\right)\right]\right|_{v_{z}=0}=0,
\end{gathered}
$$

where we used the well known Delta function properties: $\int_{-\infty}^{+\infty} g(x) \delta(x-a) \mathrm{d} x=g(a)$ and $\int_{-\infty}^{+\infty} g(x) \dot{\delta}(x-a) \mathrm{d} x=-\dot{g}(a)$. The remaining projections can be calculated in a similar way.

\section{Appendix B: Two dimensional $L U$ decomposition}

The $L U$ decomposition for the two dimensional version of the modified Stone method presented in Ujevic \& Letelier (2005) is,

$$
\begin{aligned}
L_{D B}^{p}= & \frac{A_{D B}^{p}}{1+\alpha\left[U_{N}^{p-\left(n_{i j k}+n_{i j}\right)}+U_{E}^{p-\left(n_{i j k}+n_{i j}\right)}+U_{U B}^{p-\left(n_{i j k}+n_{i j}\right)}\right]}, \\
L_{D}^{p}= & \frac{A_{D}^{p}-L_{D B}^{p} U_{T}^{p-\left(n_{i j k}+n_{i j}\right)}}{1+\alpha\left[U_{N}^{p-n_{i j k}}+U_{E}^{p-n_{i j k}}\right]}, \\
L_{D T}^{p}= & \left(A_{D T}^{p}-L_{D}^{p} U_{T}^{p-n_{i j k}}\right) /\left\{1+\alpha\left[U_{N}^{p-\left(n_{i j k}-n_{i j}\right)}\right.\right. \\
& \left.\left.+U_{E}^{p-\left(n_{i j k}-n_{i j}\right)}+U_{T}^{p-\left(n_{i j k}-n_{i j}\right)}+U_{U T}^{p-\left(n_{i j k}-n_{i j}\right)}\right]\right\} \\
L_{B}^{p}= & \frac{A_{B}^{p}-L_{D}^{p} U_{U B}^{p-n_{i j k}}-L_{D B}^{p} U_{U}^{p-\left(n_{i j k}+n_{i j}\right)}}{1+\alpha\left[U_{N}^{p-n_{i j}}+U_{E}^{p-n_{i j}}+U_{U B}^{p-n_{i j}}\right]} \\
L_{W}^{p}= & \frac{A_{W}^{p}}{1+\alpha\left[U_{N}^{p-n_{j}}+U_{T}^{p-n_{j}}+U_{U B}^{p-n_{j}}+U_{U}^{p-n_{j}}+U_{U T}^{p-n_{j}}\right]}
\end{aligned}
$$


Table B.1. Relations and nomenclature between the matrix form and the one dimensional storage index at node $p$ of the thirteen terms used in the discretization of the Fokker-Planck equation.

\begin{tabular}{cccc}
\hline \hline Matrix form & Abbreviation & Name & Position from node $p$ \\
\hline & \multicolumn{2}{c}{ Basic diagonals } \\
$f(i, j, k, l)$ & $P$ & point & $p$ \\
$f(i, j+1, k, l)$ & $N$ & north & $p+1$ \\
$f(i, j-1, k, l)$ & $S$ & south & $p-1$ \\
$f(i+1, j, k, l)$ & $E$ & east & $p+n_{j}$ \\
$f(i-1, j, k, l)$ & $W$ & west & $p-n_{j}$ \\
$f(i, j, k+1, l)$ & $T$ & top & $p+n_{i j}$ \\
$f(i, j, k-1, l)$ & $B$ & bottom & $p-n_{i j}$ \\
$f(i, j, k, l+1)$ & $U$ & up & $p+n_{i j k}$ \\
$f(i, j, k, l-1)$ & $D$ & down & $p-n_{i j k}$ \\
& Mixed diagonals & \\
$f(i, j, k+1, l+1)$ & $U T$ & uptop & $p+\left(n_{i j k}+n_{i j}\right)$ \\
$f(i, j, k-1, l+1)$ & $U B$ & upbottom & $p+\left(n_{i j k}-n_{i j}\right)$ \\
$f(i, j, k+1, l-1)$ & $D T$ & downtop & $p-\left(n_{i j k}-n_{i j}\right)$ \\
$f(i, j, k-1, l-1)$ & $D B$ & downbottom & $p-\left(n_{i j k}+n_{i j}\right)$ \\
\hline
\end{tabular}

$$
\begin{aligned}
& L_{S}^{p}=\frac{A_{S}^{p}}{1+\alpha\left[U_{E}^{p-1}+U_{T}^{p-1}+U_{U B}^{p-1}+U_{U}^{p-1}+U_{U T}^{p-1}\right]}, \\
& H_{1}=\alpha\left[L_{D B}^{p} U_{N}^{p-\left(n_{i j k}+n_{i j}\right)}+L_{D}^{p} U_{N}^{p-n_{i j k}}+L_{D T}^{p} U_{N}^{p-\left(n_{i j k}-n_{i j}\right)}\right. \\
& \left.+L_{B}^{p} U_{N}^{p-n_{i j}}+L_{W}^{p} U_{N}^{p-n_{j}}\right], \\
& H_{2}=\alpha\left[L_{D B}^{p} U_{E}^{p-\left(n_{i j k}+n_{i j}\right)}+L_{D}^{p} U_{E}^{p-n_{i j k}}+L_{D T}^{p} U_{E}^{p-\left(n_{i j k}-n_{i j}\right)}\right. \\
& \left.+L_{B}^{p} U_{E}^{p-n_{i j}}+L_{S}^{p} U_{E}^{p-1}\right] \\
& H_{3}=\alpha\left[L_{D T}^{p} U_{T}^{p-\left(n_{i j k}-n_{i j}\right)}+L_{W}^{p} U_{T}^{p-n_{j}}+L_{S}^{p} U_{T}^{p-1}\right] \text {, } \\
& H_{4}=\alpha\left[L_{D B}^{p} U_{U B}^{p-\left(n_{i j k}+n_{i j}\right)}+L_{B}^{p} U_{U B}^{p-n_{i j}}+L_{W}^{p} U_{U B}^{p-n_{j}}+L_{S}^{p} U_{U B}^{p-1}\right], \\
& H_{5}=\alpha\left[L_{W}^{p} U_{U}^{p-n_{j}}+L_{S}^{p} U_{U}^{p-1}\right] \\
& H_{6}=\alpha\left[L_{D T}^{p} U_{U T}^{p-\left(n_{i j k}-n_{i j}\right)}+L_{W}^{p} U_{U T}^{p-n_{j}}+L_{S}^{p} U_{U T}^{p-1}\right], \\
& L_{P}^{p}=H_{1}+H_{2}+H_{3}+H_{4}+H_{5}+H_{6}-L_{S}^{p} U_{N}^{p-1}-L_{W}^{p} U_{E}^{p-n_{j}} \\
& -L_{B}^{p} U_{T}^{p-n_{i j}}-L_{D T}^{p} U_{U B}^{p-\left(n_{i j k}-n_{i j}\right)}-L_{D}^{p} U_{U}^{p-n_{i j k}} \\
& -L_{D B}^{p} U_{U T}^{p-\left(n_{i j k}+n_{i j}\right)}, \\
& U_{N}^{p}=\frac{A_{N}^{p}-H_{1}}{L_{P}^{p}} \\
& U_{E}^{p}=\frac{A_{E}^{p}-H_{2}}{L_{P}^{p}} \\
& U_{T}^{p}=\frac{A_{T}^{p}-H_{3}-L_{D T}^{p} U_{U}^{p-\left(n_{i j k}-n_{i j}\right)}-L_{D}^{p} U_{U T}^{p-n_{i j k}}}{L_{P}^{p}}, \\
& U_{U B}^{p}=\frac{A_{U B}^{p}-H_{4}-L_{B}^{p} U_{U}^{p-n_{i j}}}{L_{P}^{p}}, \\
& U_{U}^{p}=\frac{A_{U}^{p}-H_{5}-L_{B}^{p} U_{U T}^{p-n_{i j}}}{L_{P}^{p}}
\end{aligned}
$$$$
U_{U T}^{p}=\frac{A_{U T}^{p}-H_{6}}{L_{P}^{p}} .
$$

where $X_{Y}^{p}$ is the element $p$ of the diagonal $Y$ in matrix $X$. These relations have to be calculated in the order specified above. The notation used for the thirteen point molecule is describe in Table B.1.

\section{References}

Aarseth, S. J., \& Binney, J. 1978, MNRAS, 185, 227

Ashurov, A. E. 2004, ApJ, 127, 2154

Binney, J., \& Tremaine, S. 1987, Galactic Dynamics (New Jersey: Princeton University Press)

Chandrasekhar, S. 1939, An Introduction to the Theory of Stellar Structure (University of Chicago Press), reissued by Dover 1958, Chicago

Cohn, H. 1979, ApJ, 234, 1036

de Zeeuw, P. T. 1985, MNRAS, 216, 273

de Zeeuw, P. T., Hunter, C., \& Schwarzschild, M. 1987, ApJ, 317, 607

Eddington, A. S. 1916, MNRAS, 76, 572

Freeman, K. C. 1966, MNRAS, 134, 1

Gerald, C. F., \& Wheatley, P. O., Applied Numerical Analysis, Fifth edition (Addison-Wesley)

Hunter, C., \& de Zeeuw, P. T., 1992, ApJ, 389, 79

Jeans, J. H. 1915, MNRAS, 76, 70

Joshi, K. J., Nave, C. P., \& Rasio, F. A. 2001, ApJ, 550, 691

Kalnajs, A. J. 1972, ApJ, 175, 63

King, I. R. 1966, AJ, 71, 64

Kuzmin, G. 1956, AZh., 33, 27

Letelier, P. S. 2003, Phys. Rev. D, 68, 104002

Mestel, L. 1963, MNRAS, 126, 553

Michie, R. W. 1963, MNRAS, 125, 127

Michie, R. W., \& Bodenheimer, P. H. 1963, MNRAS, 126, 269

Miller, R. H., \& Smith, B. F. 1979a, ApJ, 227, 407

Miller, R. H., \& Smith, B. F. 1979b, ApJ, 227, 785

Miyamoto, M. 1971, PASJ, 23, 21

Ng, E. W. 1967, ApJ, 150, 787

Nishida, M. T., Watanabe, Y., Fujiwara, T., \& Kato, S. 1984, PASJ, 36,27 
Plummer, H. C. 1911, MNRAS, 71, 460

Rosenbluth, M. N., MacDonald, W. M., \& Judd, D. L. 1957, Phys. Rev., 107, 1

Schuster, A. 1883, British Assoc. Report, 427

Schwarzschild, M. 1979, ApJ, 232, 236

Statler, T. S. 1987, ApJ, 321, 113

Stone, H. L. 1968, SIAM J. Numer. Anal., 5, 530

Takahashi, K. 1995, PASJ, 47, 561

Takahashi, K., Lee, H. M., \& Inagaki, S. 1997, MNRAS, 292, 331

Teuben, P. 1987, MNRAS, 227, 815

Theuns, T. 1996, MNRAS, 279, 827

Toomre, A. 1963, ApJ, 138, 385
Ujevic, M., \& Letelier, P. S. 2004, Phys. Rev. D, 70, 084015

Ujevic, M., \& Letelier, P. S. 2005, submitted, see http://www .arxiv.org/abs/physics/0505093 van Albada, T. S. 1982, MNRAS, 201, 939

van de Ven, G., Hunter, C., Verolme, E. K., \& de Zeeuw, P. T. 2003, MNRAS, 342, 1056

Vandervoort, P. O. 1980, ApJ, 240, 478

Vogt, D., \& Letelier, P. S. 2003, Phys. Rev. D, 68, 084010

Watanabe, Y., Inagaki, S., Nishida, M. T., Tanaka, Y. D., \& Kato, S. 1981, PASJ, 33, 541

Wilkinson, A., \& James, R. A. 1982, MNRAS, 199, 171

Yepes, G., \& Domínguez-Tenreiro, R. 1992, ApJ, 387, 27 